\title{
Diel, Tidal and Vertical Variations of Phytoplankton and Its Environment in Frobisher Bay STEPHEN I.C. HSIAO ${ }^{1}$
}

(Received 3 January 1991; accepted in revised form 25 January 1992)

\begin{abstract}
Phytoplankton were collected and environmental measurements made at depths of $0,1,3,5,7,10,20$ and $30 \mathrm{~m}$ at $3 \mathrm{~h}$ intervals through a tidal cycle in the summer in Frobisher Bay. Light, temperature, salinity and nutrients varied vertically. Concentration of chlorophyll $a$, species composition, number of species and diversity also exhibited a pronounced vertical variation, while phytoplankton cell numbers and the evenness of the species distribution was uniform through the water column. Phytoplankton biomass and composition changed considerably with time. However, diel variations in species diversity and evenness were minor under the existing environmental conditions, where only light fluctuated significantly. The integrated values of biomass, nutrients and mean temperature and salinity showed neither significant diel nor tidal variations.
\end{abstract}

Key words: arctic phytoplankton, biomass, species composition, abundance, diversity, environmental factors

RÉSUMÉ. En été, on a effectué des prélèvements de phytoplancton et procédé à des mesures environnementales à des profondeurs de $0,1,3,5,7$, 10,20 et $30 \mathrm{~m}$, à des intervalles de $3 \mathrm{~h}$, durant un cycle de marée, dans la baie Frobisher. La lumière, la température, la salinité et les éléments nutritifs variaient verticalement. La teneur en chlorophylle $a$, la variété des espèces, le nombre des espèces et la diversité montraient aussi une nette variation verticale, tandis que le nombre de cellules de phytoplancton et l'uniformité de la distribution des espèces étaient les mêmes dans toute la colonne d'eau. La biomasse et la composition du phytoplancton changeaient considérablement en fonction du temps. Cependant, les variations nycthémérales dans la diversité et l'uniformité des espèces étaient minimes dans les conditions ambiantes du moment, où seule la lumière fluctuait de façon significative. Les valeurs intégrées de la biomasse, des éléments nutritifs ainsi que de la température et de la salinité moyennes ne montraient pas de variations significatives, qu'elles soient nycthémérales ou dues à la marée.

Mots clés: phytoplancton arctique, biomasse, variété des espèces, abondance, diversité, facteurs environnementaux

Traduit pour le journal par Nésida Loyer.

\section{INTRODUCTION}

Phytoplankton biomass, distribution and species composition change continuously with variations in environmental temperature (Kamykowski, 1981), light (Nelson and Brand, 1979), nutrient availability (Cullen and Horrigan, 1981), grazing pressure (Tiselius, 1988), tide and water movements (Balch, 1981; Demers et al., 1986), seasons (Hsiao, 1980, 1988) and even with time of day (Kana et al., 1985). Endogenous rhythms also affect the diel distribution patterns of phytoplankton (Sournia, 1974). Diel rhythms in nutrient uptake (Whalen and Alexander, 1984), chlorophyll synthesis (Owens et al., 1980), cell division (Nelson and Brand, 1979; Harding and Heinbokel, 1984) and photosynthetic assimilation (Legendre et al., 1988; Vandevelde et al., 1989) are well documented for natural phytoplankton populations, but relatively little is known of vertical changes in their composition (Reid et al., 1978; Venrick, 1988). These biological and environmental variables have not been measured in arctic waters in relation to fluctuations in both tides and irradiance. The present study was undertaken to examine the influence of environmental factors on the vertical distribution of phytoplankton taxa and biomass in a shallow, cold and dynamic coastal environment during diel and lunar tidal cycles.

\section{MATERIALS AND METHODS}

\section{Study Area}

Frobisher Bay is a semi-enclosed embayment in southeast Baffin Island, divided by islands into a larger outer and a smaller inner bay. Station $1\left(63^{\circ} 42.8^{\prime} \mathrm{N}, 68^{\circ} 30.8^{\prime} \mathrm{W}\right)$ (Fig. 1$)$ is located in the latter and has a depth of 34-45 m, depending on

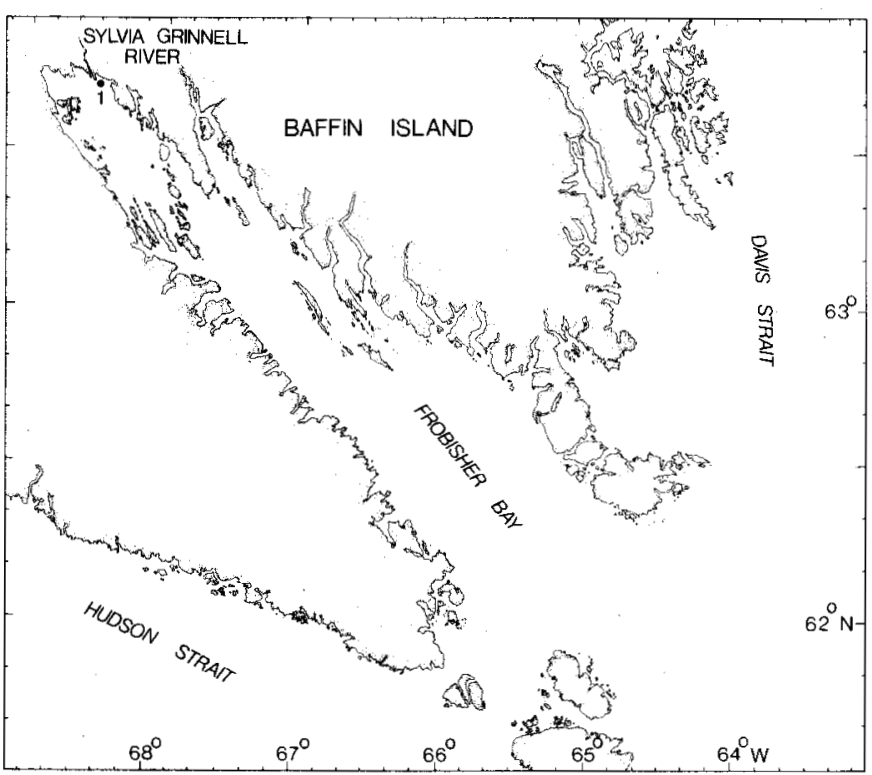

FIG. 1. Station location in Frobisher Bay.

the tide. The bay has typical semidiurnal tides with two high and two low waters (Fig. 2) in a lunar day of $24.84 \mathrm{~h}$. The maximal tidal amplitude of $11 \mathrm{~m}$ results in a movement of some $17 \mathrm{~km}^{3}$ of water into and out of the inner bay during the period of a single large tide. The mean current to and from the inner bay is about $102.8 \mathrm{~cm} \cdot \mathrm{s}^{-1}$ (Grainger, 1975). These rapidly exchanging waters are also subjected to large natural perturbation by wind, tidal mixing and river runoff from the nearby Sylvia Grinnell River.

\footnotetext{
'Department of Fisheries and Oceans, Arctic Biological Station, 555 boul. St-Pierre, Ste-Anne-de-Bellevue, Quebec, Canada H9X 3R4; present address: Biological Oceanography Division, Maurice Lamontagne Institute, Department of Fisheries and Oceans, 850 Route de la Mer, Mont-Joli, Quebec, Canada G5H 3Z4

(C) The Arctic Institute of North America
} 




FIG. 2. Schematic of a semi-diurnal tidal cycle in Frobisher Bay. Vertical bars indicate range of tidal height; horizontal bars indicate time of day; the points at the vertical and horizontal bars on the curve indicate the data collected during a lunar tidal cycle.

\section{Sampling}

By careful scheduling of sampling, biomass and taxonomic composition of phytoplankton assemblage in Frobisher Bay, together with basic oceanographic features, were obtained for approximately the same phase of the tide and time $( \pm 1 \mathrm{~h})$ of the day through a lunar tidal cycle in the summers of 1980-81 (Fig. 2).

Phytoplankton were collected at station 1 with a $5 \mathrm{~L}$ Van Dorn sampler at depths of $0,1,3,5,7,10,20$ and $30 \mathrm{~m}$ at $3 \mathrm{~h}$ intervals through floods and ebbs during two summertime spring tides (30-31 July 1980 and 16-17 August 1981). Subsamples for chlorophyll $a$, species identification and enumeration were taken from the same water bottle.

\section{Biological Measurements}

Chlorophyll $a$ was analyzed by spectrophotometric techniques (Strickland and Parsons, 1972) and calculated using the Jeffrey and Humphrey (1975) equations.

One hundred and twenty-five (125) $\mathrm{mL}$ of water containing natural populations of phytoplankton were preserved with $2.5 \mathrm{~mL}$ of $40 \%$ formaldehyde neutralized with calcium carbonate. Samples were thoroughly shaken to suspend the cells and $10 \mathrm{~mL}$ subsamples were settled in a Zeiss phytoplankton sedimentation chamber for $24 \mathrm{~h}$. The cells were enumerated in either an area equivalent to 89 microscopic fields or the entire chamber, with the aid of a Leitz inverted microscope $(500 \times)$. Subsamples of $35 \mathrm{~mL}$ were prepared using hydrogen peroxide oxidation technique (Swift, 1967) for permanent slides of cleaned diatoms to facilitate species identification with the aid of a Leitz phase-contrast compound microscope. The cells were identified to species when possible. The classification is in accordance with that followed by Silva (1980). The algal biomass was expressed as concentrations of cells as well as chlorophyll $a$.

\section{Environmental Measurements}

Environmental measurements (seawater temperature, salinity, underwater irradiance and nutrients) were made concurrently with phytoplankton collections. Seawater temperature was measured in the water bottle with a calibrated immersion thermometer. Salinity was determined with a Bissett-Berman model 6230 laboratory salinometer. Underwater irradiance was measured with an $\mathrm{Li}-185$ quantum/radiometer/photometer equipped with an Li-193 SB underwater spherical quantum sensor (Lambda Instrument Corporation, Lincoln, Nebraska). Nutrients (nitrate, phosphate and silicate) were analyzed by spectrophotometric techniques following the methods of Strickland and Parsons (1972). Water samples for ammonia were preserved with phenol in the field and kept frozen until analysis by the method of Dal Pont et al. (1974).

\section{Data Analyses}

It is assumed that all the samples came from populations with identical variances. For those variables where a preliminary examination of the data had shown deviation from normality and heterogeneity of variance, the transformation $\log _{10}(\mathrm{X}+1)$ was applied to the raw data before analysis was made (Ibanez, 1971). An analysis of variance. suggests that data sets from 1980 and 1981 were not significantly different. Values of biological and environmental data were obtained by combining both years with the means of four or more replicate samples. They were analyzed by multiway factorial analyses of variance (ANOVA). The Duncan's multiple-range test was used to test the significance of the factors being examined at a level of 0.05 using the SAS statistical package (SAS Institute, 1988). The integrated values for light and nutrients and mean values for temperature and salinity of the water column were used to study diel and tidal effects on biomass and composition. Factors found to vary significantly with depth were plotted against time and examined for vertical variation.

Dominant species are defined as those with more than $10^{4}$ cells $\cdot \mathrm{L}^{-1}$ and occurring in at least $10 \%$ of the samples. Phytoplankton diversity was determined using the ShannonWeaver diversity $\left(\mathrm{H}^{\prime}\right)$ index (Shannon and Weaver, 1949). The diversity of a community depends on the number of species and the evenness of species distribution. The evenness is then defined as $\mathrm{J}^{\prime}=\mathrm{H}^{\prime} / \log \mathrm{S}$, where $\mathrm{H}^{\prime}$ is calculated diversity and $\mathrm{S}$ is the number of observed species (Pielou, 1975).

\section{RESULTS}

\section{Underwater Irradiance}

Mean photosynthetically active radiation (PAR, 400-700 $\mathrm{nm}$ ) was at a maximum of $192 \mu \mathrm{E} \cdot \mathrm{m}^{-2} \cdot \mathrm{s}^{-1}$ at the water surface and was attenuated with increasing depth to a minimum of 2 $\mu \mathrm{E} \cdot \mathrm{m}^{-2} \cdot \mathrm{s}^{-1}$ at a depth of $30 \mathrm{~m}$ (Fig. 3A). The integrated PAR increased during the day to a peak of $2000 \mu \mathrm{E} \cdot \mathrm{m}^{-2} \cdot \mathrm{s}^{-1}$ during the afternoon and decreased to a minimum of $0.1 \mu \mathrm{E} \cdot \mathrm{m}^{-2} \cdot \mathrm{s}^{-1}$ by midnight (Fig. 4A). The PAR was not significantly affected by tides $(\mathrm{p}>0.1)$.

\section{Seawater Temperature}

Mean water temperature at the surface was $2.5^{\circ} \mathrm{C}$ and decreased with depth $(p<0.001)$ to a minimum of $-0.4^{\circ} \mathrm{C}$ at $30 \mathrm{~m}$ (Fig. 3B). The temperature did not fluctuate significantly during the sampling period $(\mathrm{p}>0.5)$, neither from low to high tides $(p>0.1)$ nor from flood to ebb ( $p>0.5)$ (Fig. 4B).

\section{Salinity}

During flood tide the salinity of surface water decreased from $32 \%$ to $24-26 \%$ at high tide (Fig. 3C), forming a density stratification layer (Fig. 3D). During ebb tide the surface salinity gradually increased to 31-32\%o at low tide (Fig. 3C). In deeper waters the salinity remained between 32 and $33 \%$ throughout the day ( $p>0.1)$ and tidal cycle ( $p>0.5$ ) (Fig. 4C). 


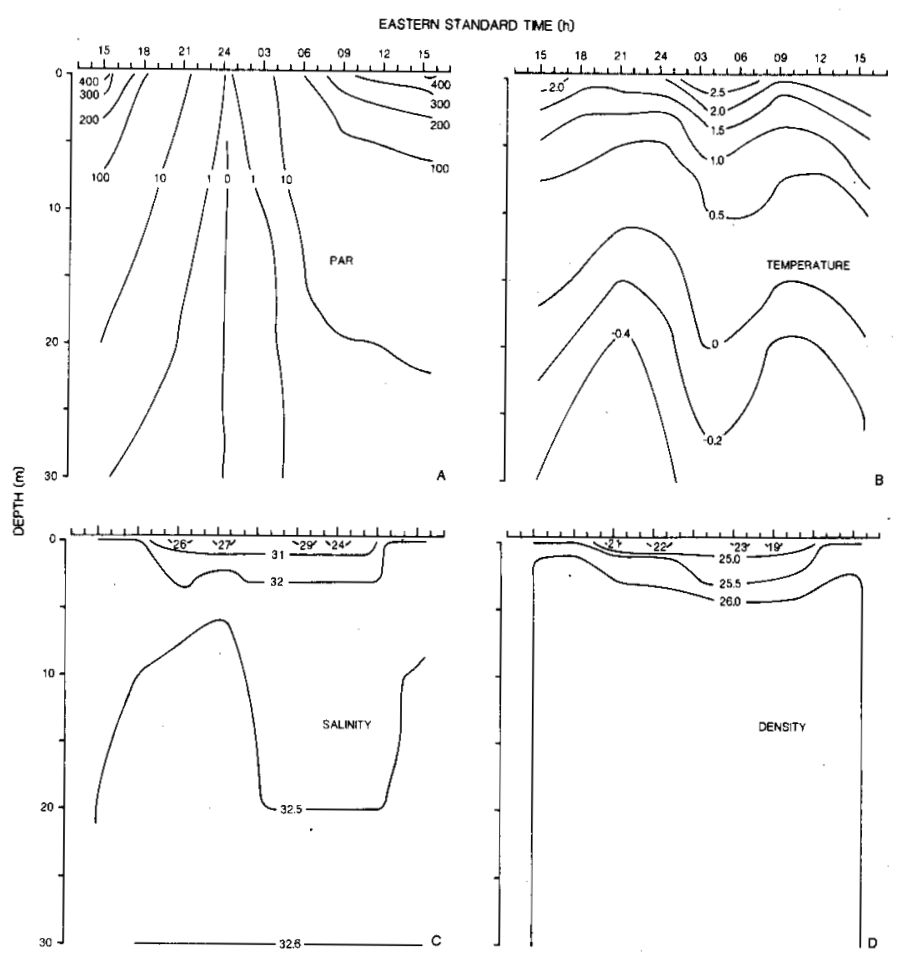

FIG. 3. Diel and vertical variations in isopleths of A) photosynthetically active radiation $\left.\left(\mu \mathrm{E} \cdot \mathrm{m}^{-2} \cdot \mathrm{s}^{-1}\right), \mathrm{B}\right)$ temperature $\left.\left({ }^{\circ} \mathrm{C}\right), \mathrm{C}\right)$ salinity $(\% o$ ), and D) density $(\sigma t)$ through a lunar tidal cycle.

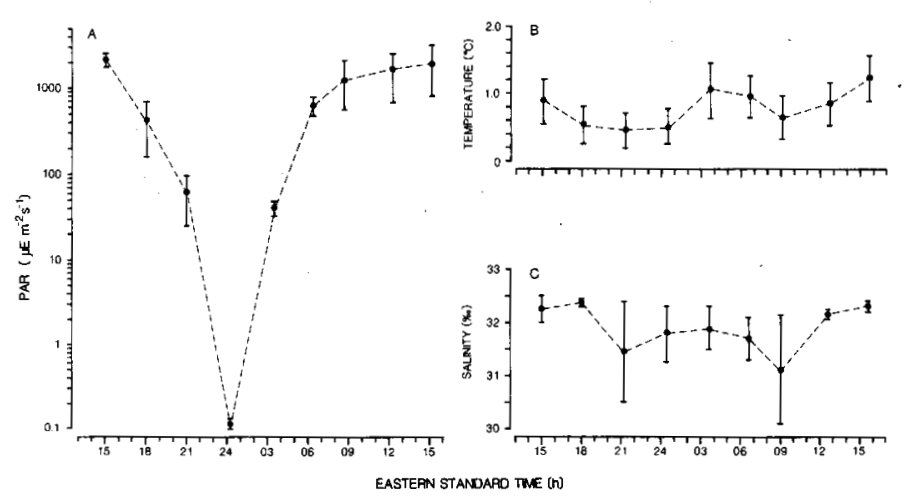

FIG. 4. Diel variations in A) integrated values of photosynthetically active radiation, B) mean temperature and C) mean salinity of the water column $(0-30 \mathrm{~m})$ through a lunar tidal cycle. Vertical bars indicate standard error.

\section{Nutrients}

Concentrations of both nitrate $(\mathrm{p}<0.05)$ and phosphate $(\mathrm{p}$ $<0.001$ ) were low in the upper $5 \mathrm{~m}$ and high in deeper water, while ammonia $(p>0.5)$ and silicate $(p>0.05)$ were distributed more uniformly throughout the water column (Fig. 5A-D). There were no significant variations in any of these nutrients (Fig. 6A-D) at any time of the day ( $p>0.1)$ or during a lunar tidal cycle $(p>0.5)$.

\section{Biomass}

The mean concentrations of chlorophyll $a$ were significantly different vertically $(\mathrm{p}<0.001)$ and were strongly influenced by both time of day $(p<0.05)$ and tidal height $(p<0.05)$. They increased with depth to $20 \mathrm{~m}$ and then decreased in deeper

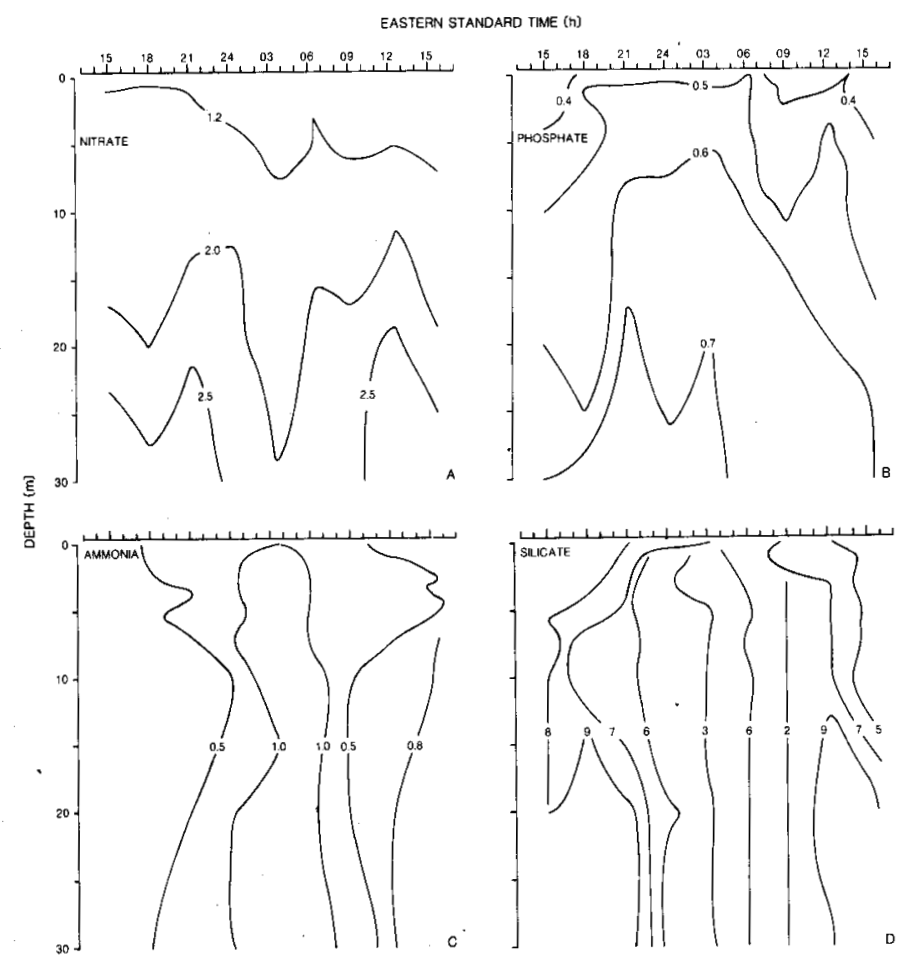

FIG. 5. Diel and vertical variations in isopleths of A) nitrate, B) phosphate, C) ammonia and D) silicate $\left(\mathrm{mg}-\mathrm{at} \cdot \mathrm{m}^{-3}\right)$ through a lunar tidal cycle.

water (Fig. 7A). Concentrations were low during low tides and high during high tides and reached a maximum at about $0900 \mathrm{~h}$. Thereafter, they decreased through the afternoon and night during flood and ebb tides. In contrast, mean phytoplankton counts did not vary significantly with depth $(p>0.5)$ or tidal height $(p>0.1)$ but varied with time of day $(p<0.05)$. Abundance was greatest in the morning during high tide just before the ebb and lowest during the ebb tide at night (Fig. 7B). Chlorophyll $a$ and cell counts (Fig. 8) integrated over the water column did not fluctuate with time of day $(p>0.5)$ or state of the tide $(\mathrm{p}>0.5)$.

\section{Composition, Abundance and Diversity}

A total of 146 phytoplankton taxa were identified (Table 1). They consisted of diatoms (centrics 27 , pennates 76 ), dinoflagellates (13), green algae (12), chrysophytes (11), euglenoids (4) and blue-green algae (3). The largest numbers of species were from the genera Navicula (15), Chaetoceros (12) and Nitzschia (9). The occurrence of dominant species at various times of day, depths and tidal heights in a lunar tidal cycle are indicated by an asterisk in Table 1 . The diatoms were the most abundant group, consisting of 11 centrics and 8 pennates. The centric diatoms comprised 5 species of Chaetoceros and 3 species each of Thalassiosira and Melosira, while pennate diatoms included 3 species of Nitzschia, 2 species of Cocconeis and one species each of Thalassiothrix, Navicula and Fragillaria. Centric species usually occurred in the upper 10 $m$ from afternoon through the night until morning during the tide above median height. Pennates were more common in the deeper waters from afternoon to midnight during the tide below median height. The green algae were represented by Carteria cordiformis at the surface in the morning and Chlamydomonas pulsatilla near the bottom $(30 \mathrm{~m})$ around midnight. 

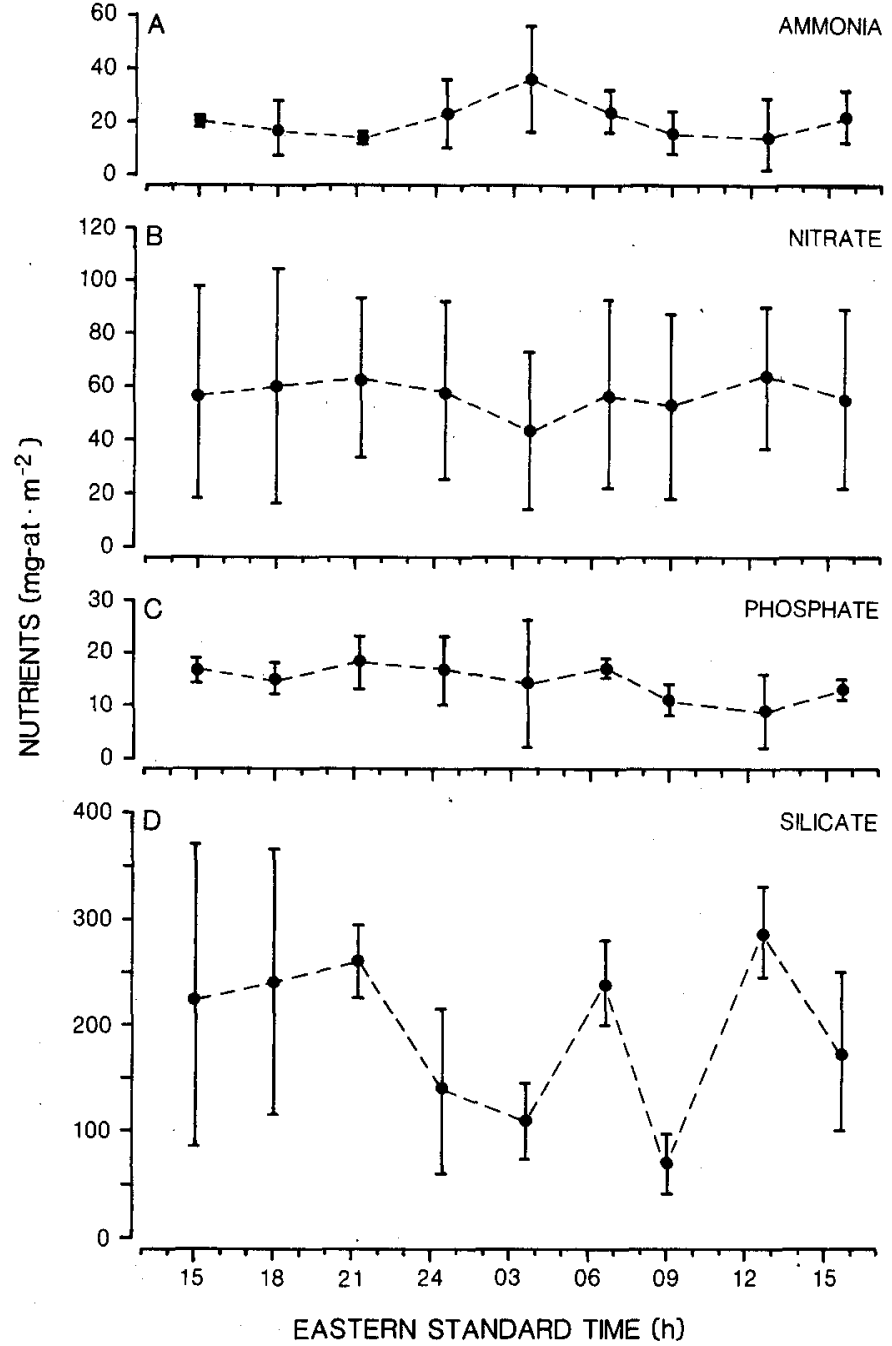

FIG. 6. Diel variations (mean $\pm \mathrm{SE}$ ) in integrated values of $\mathrm{A}$ ) ammonia, $\mathrm{B}$ ) nitrate, C) phosphate and D) silicate of the water column $(0-30 \mathrm{~m})$ through a lunar tidal cycle.

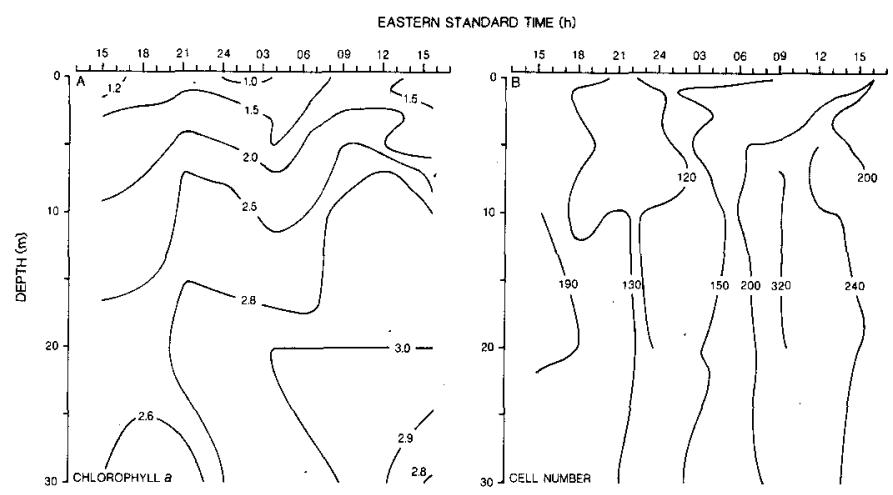

FIG. 7. Diel and vertical variations in isopleths of A) chlorophyll $a\left(\mathrm{mg} \cdot \mathrm{m}^{-3}\right)$ and $B$ ) cell number $\left(\times 10^{6}\right.$ cells $\left.\cdot \mathrm{m}^{-3}\right)$ through a lunar tidal cycle.

Both species dominated during median tide. The haptophyte, Coccolithus sp., bloomed in the lower layer of the water column during low tide in the afternoon, while Emiliania huxleyi dominated in the upper layer during median tide at night. Dinoflagellates, blue-green algae and euglenoids were never dominant.
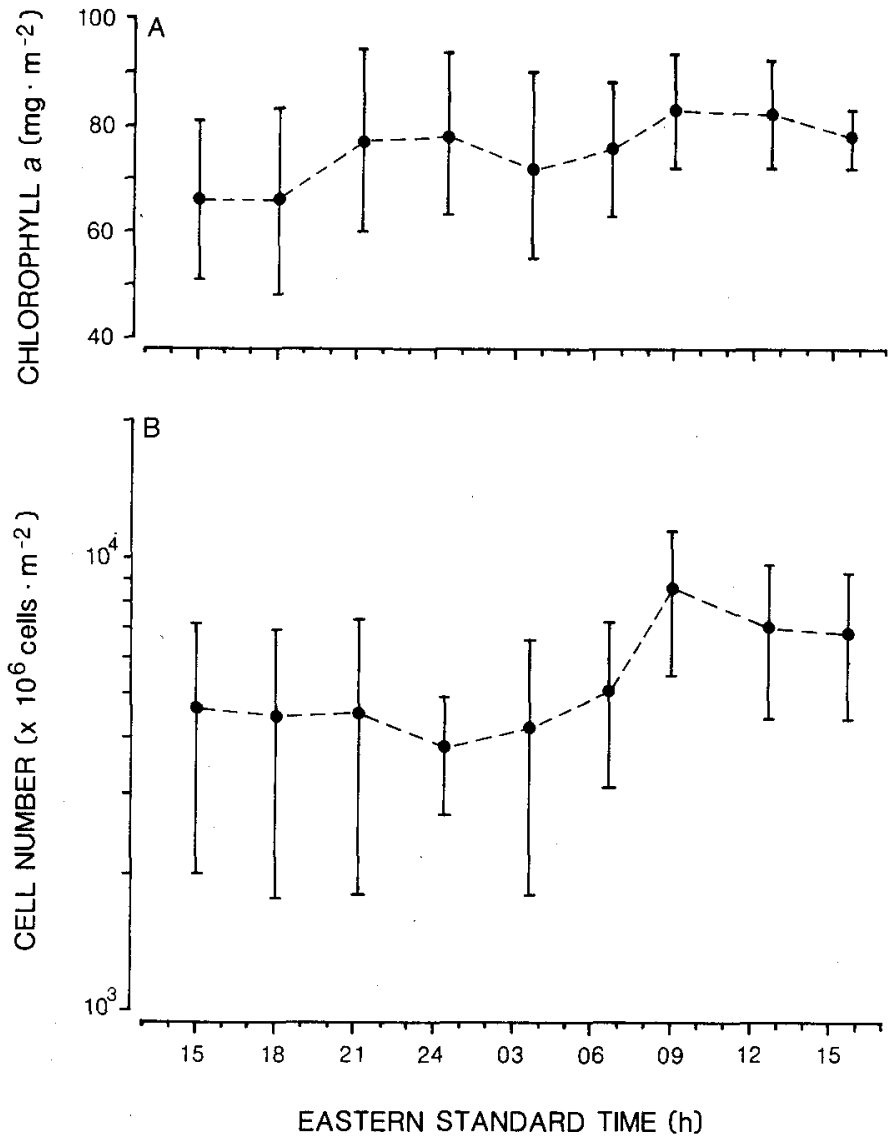

FIG. 8. Diel variations (mean $\pm \mathrm{SE}$ ) in integrated values of A) chlorophyll $a$ and B) cell numbers of the water column $(0-30 \mathrm{~m})$ through a lunar tidal cycle.

Phytoplankton composition was numerically dominated by diatoms, while dinoflagellates, chrysophytes, green algae, euglenoids and blue-green algae were sparse (Fig. 9A-F). Cell density of diatoms (Fig. 9A) was generally one or two orders of magnitude greater than that of other groups (Fig. 9B-F). Among diatoms, centrics (Fig. 10A) were always more abundant than pennates (Fig. 10B) at all depths, times of day and tidal phases. Centrics had the highest density at depths between 5 and $20 \mathrm{~m}$ in the morning at high tide just before the ebb, while pennates seemed not to vary in relation to the diel tidal rhythm. Dinoflagellates (Fig. 9B) at $10 \mathrm{~m}$ and green algae in the upper $5 \mathrm{~m}$ (Fig. 9C) had similar diel and tidal patterns as the centrics, but with 20-30 times lower cell density. Chrysophytes (Fig. 9D) had high cell density only between 10 and $20 \mathrm{~m}$ in the afternoon during low tide. Euglenoids (Fig. 9E) were generally present in low numbers and aggregated in the upper $10 \mathrm{~m}$. Their abundance did not significantly vary with time or tides. Blue-green algae (Fig. 9F) were the least abundant of the phytoplankton groups. They did not exhibit any significant patterns except for some slight evidence of patchiness.

Species diversity (Fig. 11) was significantly higher at the surface than in the deeper water $(p<0.05)$ but did not fluctuate with time of day $(p>0.1)$ or with tides $(p>0.5)$. The components of diversity, i.e., the number of species and the evenness, showed a relationship between the distribution of species and the environment. The number of species (Fig. 12A) was significantly lower in the deeper waters $(p<0.005)$, while the evenness (Fig. 12B) was unaffected by depth $(\mathrm{p}>0.05)$. 
TABLE 1. Phytoplankton species and their mean maximum occurrence in samples taken during a lunar tidal cycle

\begin{tabular}{|c|c|c|c|c|c|c|c|c|c|c|c|}
\hline & $\begin{array}{c}\text { Density } \\
\left(\times 10^{3} \text { cells } \cdot \mathrm{L}^{-1}\right) \\
\end{array}$ & $\begin{array}{c}\text { Abundance } \\
\text { (\% of } \\
\text { sample) }\end{array}$ & $\begin{array}{l}\text { Time** } \\
\text { (EST) }\end{array}$ & $\begin{array}{c}\text { Tidal } \\
\text { height } \\
\text { (m) }\end{array}$ & $\begin{array}{l}\text { Sampling } \\
\text { depth } \\
\text { (m) }\end{array}$ & & $\begin{array}{c}\text { Density } \\
\left(\times 10^{3} \text { cells } \cdot \mathrm{L}^{-1}\right)\end{array}$ & $\begin{array}{c}\text { Abundance } \\
\text { (\% of } \\
\text { sample) }\end{array}$ & $\begin{array}{l}\text { Time** }^{*} \\
(\mathrm{EST})\end{array}$ & $\begin{array}{c}\text { Tidal } \\
\text { height } \\
(\mathrm{m})\end{array}$ & $\begin{array}{c}\text { Sampling } \\
\text { depth } \\
\text { (m) }\end{array}$ \\
\hline Bacillariophyceae & & & & & & C. scutellum var. parva & & & & & \\
\hline Centrales & & & & & & (Grunow) Cleve & 0.1 & 0.1 & 0414 & 1.0 & 3 \\
\hline $\begin{array}{l}\text { Cerataulus turqidus } \\
\text { (Ehrenberg) }\end{array}$ & & & & & . & $\begin{array}{l}\text { C. thumensis Mayer } \\
\text { Cylindrotheca closter- }\end{array}$ & 7.3 & 3.0 & 0600 & 5.6 & 1 \\
\hline Ehrenberg & 3.6 & 1.8 & 1430 & 1.5 & 3 & ium (Ehrenberg) & & & & & \\
\hline *Chaetoceros affinis & & & & & & Reimann et Lewin & 10.9 & 5.5 & 0310 & 1.1 & 3 \\
\hline Lauder & 65.3 & 12.0 & 0600 & 5.6 & 10 & Cymbella affinis & & & & & \\
\hline${ }^{*}$ C. borealis J.W. Bailey & 29.0 & 10.8 & 0600 & 5.6 & 7 & Kützing & 18.1 & 4.4 & 0600 & 5.6 & 20 \\
\hline${ }^{*}$ C. decipiens Cleve & 94.3 & 21.7 & 0600 & 5.6 & 10 & C. leptoceros & & & & & \\
\hline $\begin{array}{l}\text { C. decipiens } \mathrm{f} \text {. } \\
\text { singularis Gran }\end{array}$ & 21.8 & 8.3 & $2348^{\circ}$ & 5.8 & 7 & $\begin{array}{l}\text { (Ehrenberg) Kützing } \\
\text { Diploneis incurvata }\end{array}$ & 29.0 & 7.9 & 0600 & 5.6 & 5 \\
\hline $\begin{array}{l}\text { C. difficilis Cleve } \\
{ }^{*} \text { C. furcellatus J.W. }\end{array}$ & 10.9 & 5.6 & 2042 & 10.6 & 10 & $\begin{array}{l}\text { (Gregory) Cleve } \\
\text { D. smithii (Brébisson) }\end{array}$ & 0.2 & 0.4 & 1315 & 5.8 & 0 \\
\hline Bailey & 90.7 & 15.3 & 0600 & 5.6 & 7 & Cleve & 3.6 & 0.4 & 0600 & 5.6 & 1 \\
\hline C. ingolfianus & & & & & & D. stroemi Hustedt & 3.6 & 1.5 & 2348 & 5.8 & 1 \\
\hline Ostenfeld & 14.5 & 8.5 & 1430 & 1.5 & 0 & Eunotia denticulata & & & & & \\
\hline C. lauderi Ralfs & 1.1 & 0.8 & 0924 & 10.7 & 10 & (Brébisson) & & & 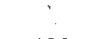 & & \\
\hline C. septentrionalis & & & & & & Rabenhorst & 3.6 & 2.0 & 1430 & 1.5 & 1 \\
\hline Oestrup & 7.3 & 9.1 & 1430 & 1.5 & 7 & E. exigua (Brébisson) & & & & & \\
\hline C. socialis Lauder & 0.1 & 0.1 & 1615 & 1.0 & 5 & Cleve & 7.3 & 3.5 & 1730 & 6.0 & 7 \\
\hline${ }^{*}$ C. tortissimus Gran & 36.3 & 14.9 & 2348 & 5.8 & 1 & E. serra var. diadema & & & & & \\
\hline $\begin{array}{l}\text { C. wighami } \text { Brightwell } \\
\text { Coscinodiscus }\end{array}$ & 43.5 & 7.4 & 0600 & 5.6 & 7 & $\begin{array}{l}\text { (Ehrenberg) Patrick } \\
\text { E. veneris (Kützing) }\end{array}$ & 7.3 & 4.4 & 1730 & 6.0 & 5 \\
\hline Ehrenberg & 0.1 & 0.2 & 2142 & 11.0 & 5 & De Toni & 32.6 & 8.9 & 0600 & 5.6 & 5 \\
\hline $\begin{array}{l}\text { Cyclotella spp. Kützing } \\
\text { Ditylum brightwellii }\end{array}$ & 10.9 & 3.5 & 1730 & 6.0 & 10 & $\begin{array}{l}\text { Fragilaria sp. Lungbye } \\
F \text {. construens }\end{array}$ & 3.6 & 1.3 & 1430 & 1.5 & 20 \\
\hline (T. West) Grunow & 3.6 & 1.4 & 0310 & 1.1 & 7 & (Ehrenberg) Grunow & 7.3 & 3.6 & 1430 & 1.5 & 10 \\
\hline Melosira sp. Agardh & 10.9 & 3.7 & 1430 & 1.5 & 0 & F. islandica Grunow & 2.1 & 1.7 & 0924 & 10.7 & 20 \\
\hline M. arctica (Ehrenberg) & & & & & & ${ }^{*} F$. pinnata Ehrenberg & 21.8 & 10.9 & 1430 & 1.5 & 5 \\
\hline Dickie & 21.8 & 9.4 & 2042 & 10.6 & 10 & Gomphonema spp. & & & & & \\
\hline *M. distans (Ehrenberg) & & & & & & Agardh & 3.6 & 1.8 & 1430 & 1.5 & 3 \\
\hline Kützing & 21.8 & 10.2 & 1730 & 6.0 & 3 & Grammatophora & & & & & \\
\hline${ }^{*} M$. islandica $\mathrm{O}$. Muller & 79.8 & 28.9 & 2042 & 10.6 & 7 & serpentina (Ralfs) & & & & & \\
\hline M. italica (Ehrenberg) & & & & & & Ehrenberg & 0.1 & 0.2 & 0048 & 6.0 & 1 \\
\hline Kützing & 7.3 & 3.6 & 1730 & 6.0 & 1 & Licmophora sp. Agardh & 0.1 & 0.1 & 1830 & 5.6 & 1 \\
\hline${ }^{*} M$. roseana Rabenhorst & 14.5 & 10.7 & 2348 & 5.8 & 10 & L. dalmatica (Kützing) & & & & & \\
\hline M. sulcata (Ehrenberg) & & & & & & Grunow & 0.1 & 0.2 & 0414 & 1.0 & 0 \\
\hline Kützing & 0.4 & 0.6 & 1315 & 5.8 & 7 & Navicula Bory & 3.6 & 2.1 & 1730 & 6.0 & 30 \\
\hline Thalassiosira spp. & & & & & & N. agrestis Hustedt & 14.5 & 7.3 & 0600 & 5.6 & 3 \\
\hline $\begin{array}{l}\text { Cleve } \\
* T \text {. decipiens (Grunow) }\end{array}$ & 7.3 & 3.2 & 2042 & 10.6 & 0 & $\begin{array}{l}\text { N. capitata Ehrenberg } \\
\text { N. digitoradiata }\end{array}$ & 3.6 & 3.0 & 1430 & 1.5 & 30 \\
\hline E. Jørgensen & 72.5 & 17.2 & 1140 & 5.8 & 10 & (Gregory) Ralfs & 7.3 & 2.1 & 1430 & 1.5 & 10 \\
\hline${ }^{*} T$. gravida Cleve & 21.8 & 11.8 & 2348 & 5.8 & 5 & N. forcipata Greville & 0.1 & 0.2 & 1315 & 5.8 & 3 \\
\hline${ }^{* T}$ T. nordenskioeldii & & & & & & N. gelida Grunow & 7.3 & 3.6 & 1730 & 6.0 & 1. \\
\hline Cleve & 87.0 & 14.7 & 0600 & 5.6 & 7 & $N$. granii (E. & & & & & \\
\hline Pennales & & & & & & Jørgensen) Gran & 39.9 & 9.6 & 1500 & 1.2 & 20 \\
\hline Achnanthes delicatula & & & & & & N. humerosa Brébisson & 3.6 & 1.0 & 0600 & 5.6 & 5 \\
\hline (Kützing) Grunow & 3.6 & 1.3 & 1430 & 1.5 & 20 & N. imperfecta Cleve & 29.0 & 8.5 & 0600 & 5.6 & 7 \\
\hline A. kriegeri Krasske & 21.8 & 7.9 & 2042 & 10.6 & 7 & N. kariana Grunow & 3.6 & 0.8 & 1140 & 5.8 & 20 \\
\hline A. marginulata Grunow & 7.3 & 3.9 & 2348 & 5.8 & 5 & $N$. peregrina & & & & & \\
\hline Amphiprora spp. & & & & & & (Ehrenberg) Kützing & 21.8 & 7.1 & 1730 & 6.0 & 20 \\
\hline Ehrenberg & 3.6 & 1.8 & 1430 & 1.5 & 3 & * $N$. radiosa var. tenella & & & & & \\
\hline A. concilians Cleve & 0.3 & 0.8 & 0706 & 5.2 & 20 & (Brébisson) Cleve et & & & & & \\
\hline Amphora inelegans & & & & & & Möller & 32.6 & 11.0 & 1430 & 1.5 & 0 \\
\hline Cleve et Grove & 3.6 & 1.6 & 2042 & 10.6 & 0 & N. salinarum Grunow & 10.9 & 6.3 & 1730 & 6.0 & 0 \\
\hline A. laevis var. & & & & & & N. transitans var. derasa & & & & & \\
\hline $\begin{array}{l}\text { laevissima } \\
\text { (Gregory) }\end{array}$ & 7.3 & 2.0 & 1430 & 1.2 & 0 & $\begin{array}{c}\text { (Grunow) Cleve } \\
N . \text { valida Cleve et }\end{array}$ & 0.1 & 0.6 & 0924 & 10.7 & 1 \\
\hline A. terroris Ehrenberg & 7.3 & 3.5 & 1430 & 1.5 & 3 & Grunow & 32.6 & 6.0 & 0600 & 5.6 & 10 \\
\hline Cocconeis sp. & & & & & & Nitzschia spp. Hassall & 3.6 & 1.2 & 1430 & 1.5 & 0 \\
\hline Ehrenberg & 3.6 & 1.2 & 2042 & 10.6 & 3 & ${ }^{*} N$. angularis var. affinis & & & & & \\
\hline C. californica & & & & & & (Grunow) Grunow & 21.8 & 12.0 & 1430 & 1.5 & 1 \\
\hline (Grunow) Grunow & 3.6 & 1.4 & 0310 & 1.1 & 1 & ${ }^{*} N$. angustata Grunow & 47.2 & 11.2 & 1315 & 5.8 & 10 \\
\hline${ }^{*}$ C. costata Gregory & 29.0 & 15.7 & 2348 & 5.8 & 5 & ${ }^{*} N$. cylindrus (Grunow) & & & & & \\
\hline C. decipiens Cleve & 0.1 & 0.1 & 1830 & 5.6 & 1 & Hasle & 54.4 & 17.9 & 1730 & 6.0 & 20 \\
\hline $\begin{array}{l}{ }^{*} \text { C. distans Gregory } \\
\text { C. pseudomarginata }\end{array}$ & 65.3 & 21.4 & 1730 & 6.0 & 20 & $\begin{array}{l}N . \text { frigida Grunow } \\
N . \text { frustulum (Kützing) }\end{array}$ & 21.8 & 7.3 & 1430 & 1.5 & 0 \\
\hline Gregory & 3.6 & 1.9 & 0310 & 1.1 & 30 & Grunow & 7.3 & 4.4 & 1730 & 6.0 & 5 \\
\hline C. scutellum Ehrenberg & 10.9 & 5.9 & 2348 & 5.8 & 30 & & & & & & ontinued) \\
\hline
\end{tabular}


TABLE 1. (Continued)

\begin{tabular}{|c|c|c|c|c|c|c|c|c|c|c|c|}
\hline & $\begin{array}{c}\text { Density } \\
\left(\times 10^{3} \text { cells } \cdot \mathrm{L}^{-1}\right)\end{array}$ & $\begin{array}{l}\text { Abundance } \\
\text { (\% of } \\
\text {. sample) }\end{array}$ & $\begin{array}{l}\text { Time** } \\
\text { (EST) }\end{array}$ & $\begin{array}{l}\text { Tidal } \\
\text { height } \\
\text { (m) }\end{array}$ & $\begin{array}{l}\text { Sampling } \\
\text { depth } \\
\text { (m) }\end{array}$ & & $\begin{array}{c}\text { Density } \\
\left(\times 10^{3} \text { cells } \mathrm{L}^{-1}\right)\end{array}$ & $\begin{array}{l}\text { Abundance } \\
\text { (\% of } \\
\text { sample) }\end{array}$ & $\begin{array}{l}\text { Time** } \\
\text { (EST) }\end{array}$ & $\begin{array}{l}\text { Tidal } \\
\text { height } \\
(\mathrm{m})\end{array}$ & $\begin{array}{c}\text { Sampling } \\
\text { depth } \\
\text { (m) }\end{array}$ \\
\hline N. hungarica Grunow & 7.3 & 2.1 & 1430 & 1.5 & 10 & Trochiscia multispinosa & & & & & \\
\hline N. seriata Cleve & 0.3 & 0.3 & 0414 & 1.0 & 3 & (Moebius) & & & & & \\
\hline N. sigma (Kützing) & & & & & & Lemmermann & 4.0 & 6.4 & 2142 & 11.0 & 1 \\
\hline Wm. Smith & 0.6 & 1.0 & 1530 & 1.1 & 3 & Chrysophyceae & & & & & \\
\hline Opephora martyi & & & & & & Dinobryon spp. & & & & & \\
\hline $\begin{array}{l}\text { Héribaud } \\
\text { Pinnularia } \mathrm{sp} \text {. }\end{array}$ & 21.8 & 7.8 & 2042 & 10.6 & 5 & $\begin{array}{l}\text { Ehrenberg } \\
\text { D. balticum (Schütt) }\end{array}$ & 7.0 & 9.5 & 1830 & 5.6 & 1 \\
\hline Ehrenberg & 0.1 & 0.1 & 0048 & 6.0 & 10 & Lemmermann & 0.1 & 0.1 & 1830 & 5.6 & 1 \\
\hline $\begin{array}{l}\text { P. interrupta f. biceps } \\
\text { (Gregory) Cleve }\end{array}$ & 0.1 & 0.1 & 1315 & 5.8 & 30 & $\begin{array}{l}\text { Isochrysis sp. Parke } \\
\text { Ophiocytium sp. }\end{array}$ & 0.1 & 0.1 & 0924 & 10.7 & 7 \\
\hline $\begin{array}{l}\text { P. quadratarea var. } \\
\text { stuxbergii (Cleve) }\end{array}$ & & & & & & $\begin{array}{l}\text { Nägeli } \\
\text { Synura uvella }\end{array}$ & 7.3 & 2.4 & 1430 & 1.5 & 0 \\
\hline $\begin{array}{l}\text { Cleve } \\
P . \text { viridis (Nitzsch) }\end{array}$ & 3.6 & 0.9 & 0600 & 5.6 & 1 & $\begin{array}{l}\text { Ehrenberg } \\
\text { Cryptophyceae }\end{array}$ & 0.6 & 2.0 & 1830 & 5.6 & 3 \\
\hline Ehrenberg & 3.6 & 1.4 & 0310 & 1.1 & 20 & Hemiselmis rufescens & & & & & \\
\hline $\begin{array}{l}\text { Plagiogramma } \\
\text { staurophorum }\end{array}$ & & & & & & $\begin{array}{r}\text { Parke } \\
\text { Cyanophyceae }\end{array}$ & 0.1 & 0.1 & 0924 & 10.7 & 7 \\
\hline (Gregory) Heiberg & 0.1 & 0.2 & 1530 & 1.0 & 0 & Anabaena sp. & & & & & \\
\hline Pleurosigma angulatum & & & & & & St. Vincent & 0.1 & 0.1 & 2142 & 11.0 & 20 \\
\hline $\begin{array}{l}\text { (Quekett) Wm. Smith } \\
\text { P. stuxbergii Cleve et }\end{array}$ & 0.1 & 0.2 & 1315 & 5.8 & 10 & $\begin{array}{l}\text { Lyngbya aestuarii } \\
\text { (Mert) Liebman }\end{array}$ & 0.1 & 0.2 & 1315 & 5.8 & 3 \\
\hline $\begin{array}{l}\text { Grunow } \\
\text { Rhabdonema arcuatum }\end{array}$ & 3.6 & 1.1 & 1430 & 1.5 & 0 & $\begin{array}{l}\text { Spirulina sp. Turpin } \\
\text { Dictyochophyceae }\end{array}$ & 0.1 & 0.2 & 2142 & 11.0 & 1 \\
\hline (Lyngbye) Kützing & 0.6 & 1.0 & 1615 & 1.0 & 1 & Distephanus speculum & & & & & \\
\hline R. minutum Kützing & 0.1 & 0.3 & 1830 & 5.6 & 10 & (Ehrenberg) Haeckel & 7.3 & 7.1 & 1430 & 1.5 & 30 \\
\hline Stauroneis quadripedis & & & & & & Dinophyceae & & & & & \\
\hline $\begin{array}{l}\text { (Cleve-Euler) } \\
\text { Hendey }\end{array}$ & 21.8 & 5.0 & 1140 & 5.8 & 20 & $\begin{array}{l}\text { Ceratium spp. Schrank } \\
\text { Dinophysis acuminata }\end{array}$ & 3.6 & 1.2 & 1430 & 1.5 & 0 \\
\hline $\begin{array}{l}\text { Synedra spp. } \\
\text { Ehrenberg } \\
\text { S. tabulata (Agardh) }\end{array}$ & 7.3 & 2.6 & 2042 & 10.6 & 5 & $\begin{array}{l}\text { Claparède et } \\
\text { Lachmann }\end{array}$ & 0.2 & 0.2 & 1615 & 1.0 & 10 \\
\hline Kützing & 11.1 & 6.9 & 0048 & 6.0 & 30 & $\begin{array}{l}\text { Glenodinium danicum } \\
\text { Paulsen }\end{array}$ & 3.6 & 1.8 & 1430 & 1.5 & 3 \\
\hline S. tabulata var. parva & & & & & & Goniaulax spp. Diesing & 0.5 & 0.5 & 0048 & 6.0 & 10 \\
\hline (Kützing) Hustedt & 7.3 & 4.0 & 1430 & 1.5 & 1 & G. monilata Howell & 0.4 & 0.6 & 2142 & 11.0 & 1 \\
\hline Tabellaria spp. & & & & & & Peridinium spp. & & & & & \\
\hline Ehrenberg & 7.3 & 3.5 & 1730 & 6.0 & 7 & Ehrenberg & 3.6 & 2.0 & 1430 & 1.5 & 1 \\
\hline Thalassionema & & & & & & P. cerasus Paulsen & 3.6 & 2.1 & 0600 & 5.6 & 7 \\
\hline $\begin{array}{l}\text { nitzschioides } \\
\text { (Grunow) Van }\end{array}$ & & & & & & $\begin{array}{l}\text { P. diabolus Cleve } \\
\text { Prorocentrum spp. }\end{array}$ & 5.5 & 7.4 & 1830 & 5.6 & 1 \\
\hline Heurck & 3.6 & 1.5 & 2348 & 5.8 & 1 & Ehrenberg & 0.1 & 0.1 & 0048 & 6.0 & 7 \\
\hline *Thalassiothrix spp. & & & & & & P. micans Ehrenberg & 0.3 & 0.2 & 0924 & 10.7 & 10 \\
\hline Cleve et Grunow & 36.3 & 35.7 & 1430 & 1.1 & 30 & $P$. rampii Sournia & 18.1 & 7.5 & 2348 & 5.8 & 1 \\
\hline $\begin{array}{l}\text { Chlorophyceae } \\
\quad \text { * Carteria cordiformis } \\
\quad \text { (Carter) Dill }\end{array}$ & & & & & & & & & & & \\
\hline $\begin{array}{l}\text { (Carter) Dill } \\
\text { Chlamydomonas } \\
\quad \text { marina Cohn } \\
\text { *C. pulsatilla }\end{array}$ & 3.6 & 10.6 & 1500 & 1.3 & 0 & $\begin{array}{l}\text { (Paulsen) Balech } \\
\text { Euglenophyceae } \\
\text { Euglena spp. }\end{array}$ & 0.1 & 0.1 & 0924 & 10.7 & 10 \\
\hline $\begin{array}{l}\text { *C.pulsathla } \\
\text { Wohlenweber }\end{array}$ & 36.3 & 20.0 & 2348 & 5.8 & 30 & Ehrenberg & 3.6 & 1.6 & 2042 & 10.6 & 0 \\
\hline Closterium sp. Nitzsch & 0.1 & 0.2 & 0414 & 1.0 & 0 & E. deses Ehrenberg & 7.3 & 1.9 & 1140 & 5.8 & 3 \\
\hline C. lineatum Ehrenberg & 0.2 & 0.2 & 1615 & 1.0 & 10 & E. schmitzii Gojdics & 0.1 & 0.1 & 0924 & 10.7 & 7 \\
\hline Cosmarium sp. Corda & 0.1 & 0.1 & 1315 & 5.8 & 7 & E. viridis Ehrenberg & 0.6 & 0.8 & 1615 & 1.0 & 5 \\
\hline Oocystis sp. Naegeli & 0.1 & 0.2 & 0924 & 10.7 & 5 & Haptophyceae & & & & & \\
\hline $\begin{array}{l}\text { Pediastrum sp. Meyen } \\
\text { Spirogyra inflata }\end{array}$ & 3.6 & 1.6 & 2042 & 10.6 & 0 & $\begin{array}{l}\text { *Coccolithus Schwarz } \\
{ }^{*} \text { Emiliania huxleyi }\end{array}$ & 170.5 & 62.7 & 1430 & 1.5 & 20 \\
\hline (Vanch.) Kützing & 3.6 & 0.9 & 1500 & 1.2 & 10 & (Lohmann) Hay & & & & & \\
\hline $\begin{array}{l}\text { Staurastrum sp. Meyen } \\
\text { S. megacanthum }\end{array}$ & 0.1 & 0.9 & 0048 & 6.0 & 0 & $\begin{array}{l}\text { et Mohler } \\
\text { Phaeocystis pouchetii }\end{array}$ & 29.0 & 11.1 & 2348 & 5.8 & 7 \\
\hline Lundell & 0.1 & 0.2 & 0414 & 1.0 & 1 & (Hariot) Lagerheim & 0.3 & 0.8 & 0706 & 5.2 & 20 \\
\hline
\end{tabular}

*Dominant species.

**Time of maximal cell division occurred in a lunar tidal cycle.

\section{DISCUSSION}

The phytoplankton community in Frobisher Bay varies in its taxonomic composition at different times of day and tidal phases. This is probably a result of the various taxonomic groups dividing according to their preferred division time at specific depths during different tidal states or during the day or night, as shown in Table 1. Nelson and Brand (1970) found that the majority of diatom species divided primarily in the 

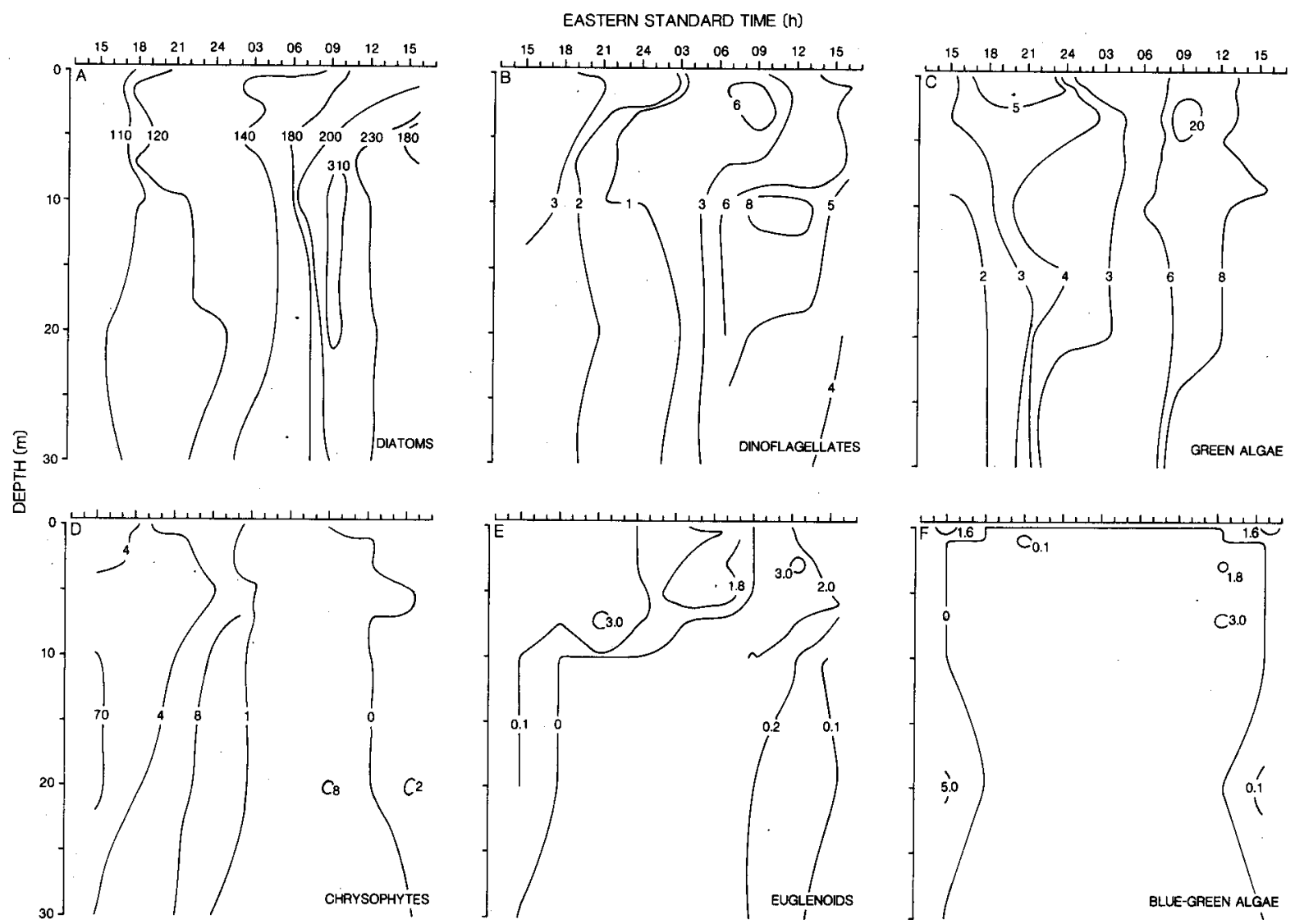

FIG. 9. Diel and vertical distribution of A) diatoms, B) dinoflagellates, C) green algae, D) chrysophytes, E) euglenoids and F) blue-green algae $\left(\times 10^{6}\right.$ cells $\mathrm{m}^{-3}$ ) through a lunar tidal cycle.

dark. Lewin and Rao (1975) showed that small groups of diatoms preferrentially divided in the light. However, Williamson (1980) demonstrated that some marine diatoms did not have preferred times for cell division. Cell division in both Chlorophyceae and Euglenophyceae were generally confined to the dark (Pirson and Lorenzen, 1966), while the peak cell division in the green flagellate Dunaliella tertiolecta occurred at the end of the light period (Eppley and Coatsworth, 1966). Chrysophytes clearly divided preferentially at night (Chisholm, 1981). In dinoflagellates, Ceratium division stages were usually restricted to late night-early morning hours (Weiler, 1980), while division of Dinophysis fortii extended through the entire day, with the maximum frequency of paired cells occurring soon after sunrise (Weiler and Chisholm, 1976).

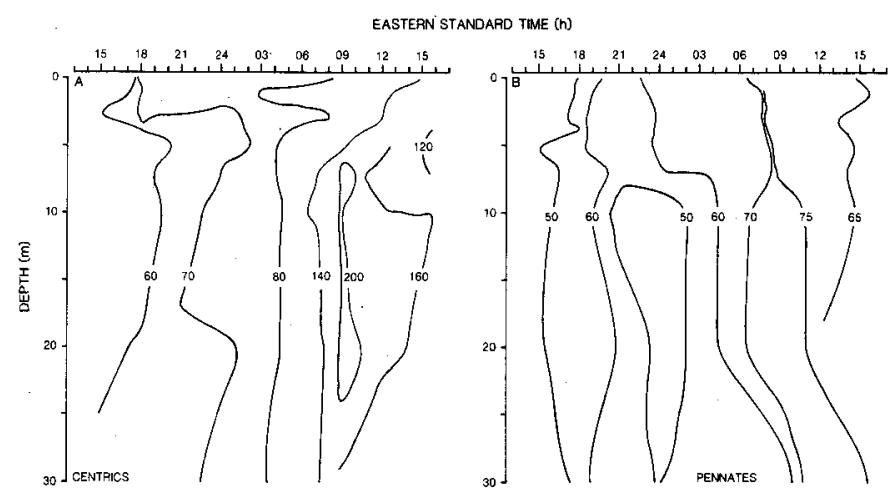

FIG. 10. Diel and vertical distribution of $A$ ) centric and B) pennate diatoms $\left(\times 10^{6}\right.$ cells $\left.\cdot \mathrm{m}^{-3}\right)$ through a lunar tidal cycle.
Sournia (1974), while indicating that the weight of evidence supported nighttime divisions by most phytoplankton, stressed that random variations could occur in areas where tides are prevalent. Later, Chisholm (1981) found that cell division of phytoplankton peaked at almost any hour of the day, depending on species and environmental conditions.

Tides have a doubly beneficial effect in upper Frobisher Bay. They not only flush in freshwater and nutrients from the nearby Sylvia Grinnell River during flooding but also generate an upward mixing, particularly when ebbing. A short period of stratified water occurred only near the surface during flood tides. The waters were subsequently mixed by the alternating tidal cycles in addition to other mixing processes, such as wind and convection (Demers et al., 1987). These significantly affect physical, chemical and biological dynamics of marine coastal environment.

The fluctuations in temperature, salinity and nutrients accompanying the tidal cycle involve more gradual transitions than do those of the day-night light cycle. Superimposed on these gradual transitions are large-amplitude, short-period oscillations in the form of waves. These can alter phytoplankton chlorophyll concentrations and cell numbers through internal tides (Haury et al., 1983; Lande and Yentsch, 1988), wind waves (Iverson et al., 1974; Demers et al., 1987), wind-driven surface currents (Harris and Trimbee, 1986), river-driven currents (Litaker et al., 1987) or horizontal tidal advection (Cloern et al., 1989). All these mixing processes occurred and were strong enough to break down the vertical stratification at the study site in Frobisher Bay. Diel variations in the abundance and distribution could also be reduced by tidally driven 


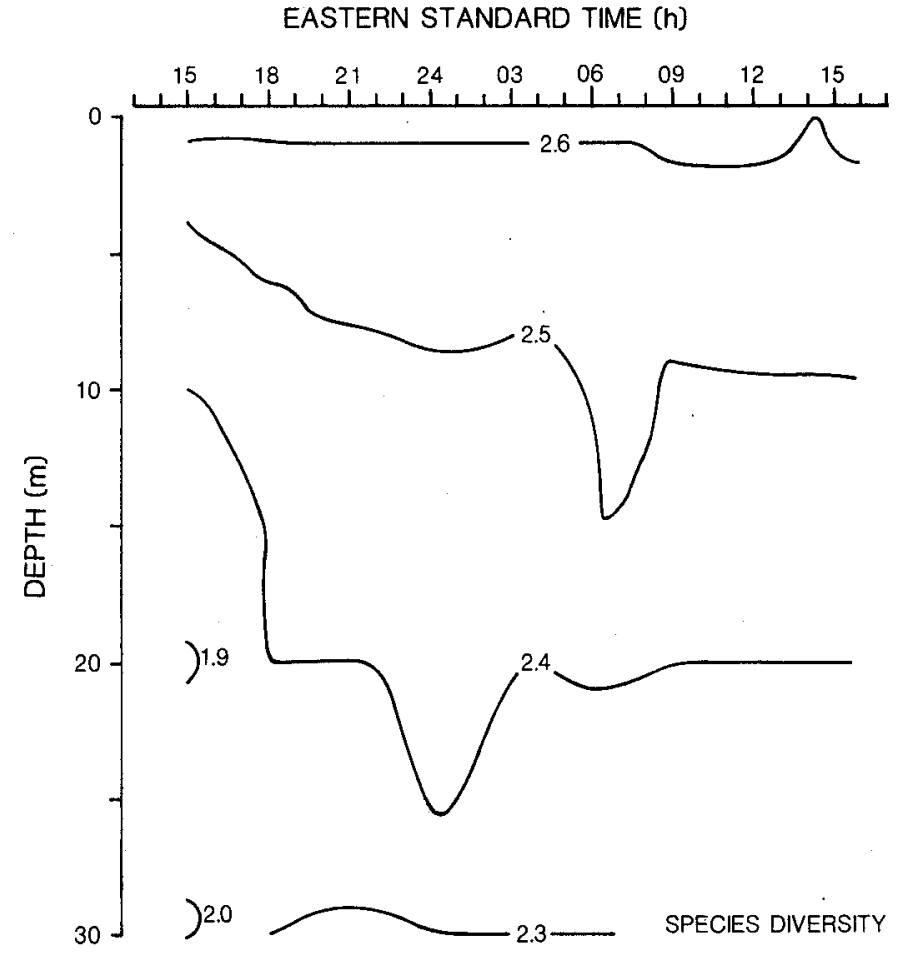

FIG. 11. Diel and vertical variations in species diversity index (bites-cell ${ }^{-1}$ ) through a lunar tidal cycle.

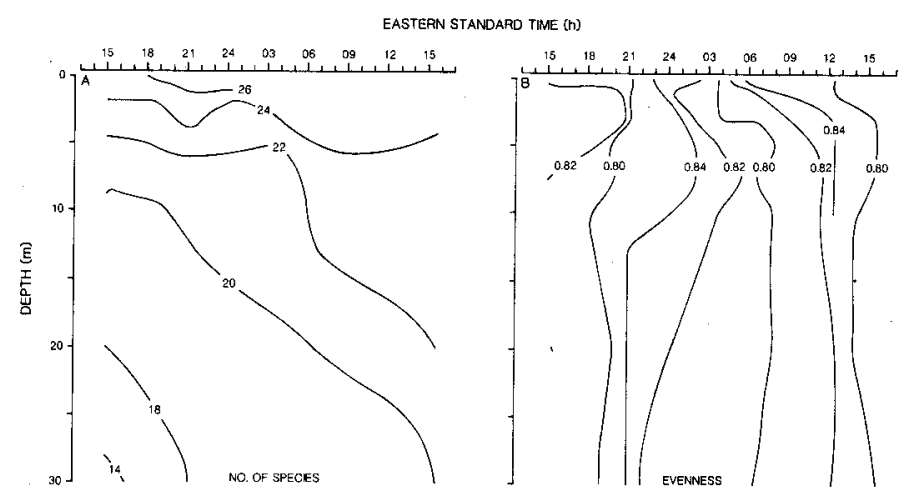

FIG. 12. Diel and vertical variations in the A) numbers of species and $B$ ) evenness through a lunar tidal cycle.

turbulence. Moreover, Hsiao (1985) found that cell division rates of phytoplankton in the same area were mostly $>24 \mathrm{~h}$ division. Species succession did not occur within a tidal cycle during summer. These were coupled with rapid mixing of the water and low zooplankton grazing. Phytoplankton cells were thus uniformly distributed through the water column by the alternation of flood and ebb tides accompanied by intense vertical and horizontal mixing.

This intense tidal mixing resuspends sediments, which not only resuspend cells but also replenish nutrients into the water column (Demers et al., 1987). These conditions may be more favorable to the growth and photosynthesis of centric diatoms that have a predominantly planktonic and photoautotrophic mode of existence in such environments (Hsiao et al., 1977; Turpin and Harrison, 1979) and exhibited maximal numbers in the morning during high tide just before ebbing. In contrast, pennate diatoms are predominantly benthic species and have heterotrophic capabilities both in the light and the dark (Hellebust and Lewin, 1977). They did not show any correlation with tidal rhythm. The other groups of phytoplankton were always less abundant than diatoms except for the haptophyte Coccolithus sp., whose cells aggregated at $20 \mathrm{~m}$ in the morning during high tide and dissipated by the afternoon ebbing tide. The green algae and dinoflagellates occurred in patches respectively at 5 and $10 \mathrm{~m}$ in the morning during the same tidal conditions with the centric diatoms. They seemed to prefer abundant light and were located in the upper layers of the water column. It has been reported that small-sized diatoms dominate in the well-mixed waters (Pingree et al., 1978; Levasseur et al., 1984), some large diatoms and microflagellates dominate in well-stratified waters (Wangersky, 1977; Levasseur et al., 1984) and dinoflagellates are more abundant at fronts between well-mixed water and more stable offshore water (Holligan, 1978) or during intervening minor spring and neap tides (Balch, 1981). However, Wangersky (1977) argued that diatom growth depended on the renewal of inorganic nutrients through tidal mixing, whereas dinoflagellates and microflagellates only became dominant when the major source of nutrients was regenerated in situ by the degradation of dissolved organic compounds by bacteria. Therefore, tidal mixing not only affects environmental conditions but also influences the growth, spatial distribution and community structure of phytoplankton in the mixed layer.

The vertical distribution of a phytoplankton assemblage was not only largely determined by the dynamics of the formation of seasonal thermocline (Kiefer and Kremer, 1981) but also associated with water column stability (Ignatiades, 1979). The depth of maximum phytoplankton production was found when the vertical stability reached a maximum as a thick thermohalocline formed (Vandevelde et al., 1987). In the physical dynamics of shallow Frobisher Bay, the taxonomic composition in the water column is probably strongly influenced by species that originated from the nearby Sylvia Grinnell River, the sea ice microalgae and/or the resuspension of bottom sediment. The change in species composition and diversity within the water column can probably be explained by difference in sinking rates (Gabric and Parslow, 1989), selective grazing (Daro, 1988) and behavioral responses to environmental conditions (Venrick, 1988), particularly to photoadaptation (Falkowski, 1983). None of the taxa was confined to a single depth but some had a depth preference. The centric diatoms, such as the dominant species of Chaetoceros, Melosira and Thalassiosira, dominated in the upper $10 \mathrm{~m}$, while the pennate diatoms Cocconeis distans, Nitzschia cylindrus and Thalassiothrix sp. dominated in deeper water. All these diatoms inhabit unstable environments and are predominant in tidally mixed coastal waters rather than in relatively stable sea ice habitats (Hsiao, 1980). The green alga Carteria cordiformis occurred in highest density near the surface, while another chlorophycean, Chlamydomonas pulsatilla, bloomed near the bottom of the water column. The haptophyte Emiliania huxleyi dominated at $7 \mathrm{~m}$, representing $11 \%$ of the population, while Coccolithus sp., representing $63 \%$, bloomed at $20 \mathrm{~m}$. The blue-green algae, dinoflagellates and euglenoids were particularly distributed in the upper water layers.

The vertical distribution of phytoplankton diversity in Frobisher Bay was highest (2.6) in the surface layer and gradually decreased with depth and became lowest (1.9) during blooming. This was largely due to in-flowing freshwater, 
which brought in more species from the nearby Sylvia Grinnell River and formed the thermohalocline. These led to active growth of the population in the mixed water, with a better nutrient balance and other physical conditions, and thus a decrease in diversity. Low diversity usually resulted from spatial or temporal predominance of a few species and unequal relative abundance. The diversity did not exhibit significant diel or tidal changes. Margalef (1977) pointed out that low diversity is associated with high primary producer/herbivore ratio and considered productivity usually inversely related to diversity in less productive waters. Diversity of phytoplankton was usually between 1 and 2.5 in coastal waters (Margalef, 1978), being especially low in blooming populations (Raymont, 1980) and physically stressed ecosystems such as in polar regions (Odum, 1971) and estuarine (Hulburt, 1963) and upwelling areas (Margalef, 1978). Values from 3.5 to 4.5 were most frequently measured in the oceanic areas (Margalef, 1978). Values might approach 5 in open tropical oceanic environments, where phytoplankton have low productivity with a large number of species. However, species diversity might be increased for most unstable environments (Reed, 1978) and later stages of succession (Ignatiades, 1969). It is clearly shown that diversity values were very closely related to species numbers, while evenness values were unimportant in determining species diversity.

The concentrations of chlorophyll $a$ varied from species to species in the populations depending on the biomass present and the conditions under which they are growing. They showed a diel periodicity, highest during the morning ebb tide and lowest during afternoon flood tide, based on the volume measurement of sample, but not on an areal basis, in the present study. This is probably because of the uniform phytoplankton density in the water column. However, chlorophyll content per cell was maximal at mid-day and minimal at night (Wood and Corcoran, 1966). Other workers described diurnal variations in chlorophyll contents ranging from an early morning minimum and afternoon maximum (Whitledge and Wirick, 1983) to the reverse, a peak in the early morning to the lowest level in the afternoon (Shimada, 1958), as well as no obvious diel pattern (Kawarada and Sano, 1968). These findings appear to be inconsistent and contradictory because of the differences in parameters measured and experimental procedures employed. Sournia (1974) concluded that the chlorophyll content of phytoplankton was subjected to a diel periodicity, independent of grazing, cell division and vertical migration. The main influence on the diel periodicity of the cellular chlorophyll content is obviously light. Natural changes in irradiance play a dominating role not only in photosynthesis, but also in inducing other circadian processes such as respiration, nutrient uptake and cell division (Soeder, 1965). Other factors such as temperature, nutrient availability, water column stability and taxonomic composition would also modify the periodicity (Owens et al., 1980; Legendre et al., 1982).

The vertical distribution of chlorophyll $a$ was characterized by a maximum in the thermohalocline and nitracline in Frobisher Bay at $20 \mathrm{~m}$, while it was independent of phytoplankton cell density. This maximum was also located at depth where irradiance was about $3 \%$ of the sea surface. It coincides with the finding of Cullen and Eppley (1981) that the chlorophyll maximum layers were generally located at a depth between 1 and $6 \%$ light levels in the vicinity of the nitracline. Chlorophyll $a$ synthesis may stop or photodegradation occur near the surface at noon on bright days (Steemann Nielsen and Jorgensen, 1962), while it may be increased in the amount per cell as a result of shade adaptation to low ambient light levels (Bienfang et al., 1983). Steele and Yentsch (1960) explained that some phytoplankters can control their vertical position and produce a chlorophyll maximum layer in the thermocline by decreased sinking rates at the pycnocline as the cells enter into the low-light and nutrient-rich waters found at the base of the euphotic zone. The concentrations of nitrate and phosphate in the water column were stratified, while ammonia and silicate were homogenous. These may be attributed to the results from a combination of preferential nutrient uptake by phytoplankton, differential growth rates of the algal populations and nutrient regeneration rates in a turbulent environment. In Frobisher Bay diatoms constitute the dominant phytoplankton. They particularly require silicon for their cell division and growth. Silicon concentration in seawater was minimum during the night, when most of diatoms divided, while the other nutrients were not significantly different at any time or phase during a tidal cycle. Lewin et al. (1966) found that silicon uptake by the diatom cells is restricted to a relatively short period following cytokinesis, when cell wall synthesis occurs. This bulk requirement is distinct from the requirement of nitrogen and phosphorus for other essential metabolic events that precede cell wall synthesis. Silicon was also regenerated at a low rate compared with nitrogen and phosphorus (Dugdale, 1972). Ammonia could be rapidly and directly utilized by marine phytoplankton (Glibert and Goldman, 1981).

In conclusion, the vertical distribution of phytoplankton in Frobisher Bay is a time-varying property influenced by physical (light, temperature, salinity and tidal mixing), chemical (nutrients) and biological (grazing and sinking rates) factors. These factors interact to produce a constant vertical profile of cell abundances. The time scale of these changes is probably controlled by the stability of the environment and the major groups of cell division. These cells respond according to the intensity and persistence of vertical mixing, since the light history of the cells is known to influence the photosynthesis-irradiance relationship by altering chlorophyll/carbon ratios (Marra and Heinemann, 1982; Falkowski, 1984; Langdon, 1988). At low light levels they can develop physiological adaptation by changes in sinking rate and cellular chlorophyll concentration in creating a subsurface chlorophyll maximum at the base of the euphotic zone and associate with the thermohalocline and nitracline. Their diversity is closely correlated with the number of species but decreased with increasing both the depth and the concentrations of nitrate and phosphate. Moreover, specific differences in sinking rate and in the ability to maintain themselves within the mixed layers may profoundly influence species composition, which is of paramount importance to the production of higher trophic levels in the marine ecosystems.

\section{ACKNOWLEDGEMENTS}

The help of many people was essential to the success of the project. In particular I wish to express my sincere thanks to J.M.A. Theriault for use of the facilities of the Iqaluit Research Laboratory, Iqaluit, N.W.T.; to Douglas Hope and J. Lovrity for field work; to J. Fife and R. Harland for computer assistance; and to Dr. J. Percy, who kindly assisted in revising the manuscript. 


\section{REFERENCES}

BALCH, W.M. 1981. An apparent lunar tidal cycle of phytoplankton blooming and community succession in the Gulf of Maine. Journal of Experimental Marine Biology and Ecology 55:65-77.

BIENFANG, P., SZYPER, J., and LAWS, E. 1983. Sinking rate and pigment responses to light-limitation of a marine diatom: Implications to dynamics of chlorophyll maximum layers. Oceanologica Acta 6:55-62.

CHISHOLM, S.W. 1981. Temporal patterns of cell division in unicellular algae. In: Platt, T., ed. Physiological bases of phytoplankton ecology. Canadian Bulletin of Fisheries and Aquatic Sciences 210:150-181.

CLOERN, J.E., POWELL, T.M., and HUZZEY, L.M. 1989. Spatial and temporal variability in south San Francisco Bay (USA). II. Temporal changes in salinity, suspended sediments, and phytoplankton biomass and productivity over tidal time scales. Estuarine, Coastal and Shelf Science 28:599613.

CULLEN, J.J., and EPPLEY, R.W. 1981. Chlorophyll maximum layers of the Southern California Bight and possible mechanisms of their formation and maintenance. Oceanologica Acta 4:23-32.

CULLEN, J.J., and HORRIGAN, S.G. 1981. Effects of nitrate on the diurnal vertical migration, carbon to nitrogen ratio, and the photosynthetic capacity of dinoflagellate Gymnodinium splendens. Marine Biology 62:81-89.

DAL PONT, G.M., HOGAN, M., and NEWELL, B. 1974. Laboratory techniques in marine chemistry II. Determination of ammonia in sea water and preservation of samples for nitrate analysis. Sydney: Commonwealth Scientific and Industrial Research Organization, Division of Fisheries and Oceanography. Report 55. 1-7.

DARO, M.H. 1988. Migratory and grazing behavior of copepods and vertical distribution of phytoplankton. Bulletin of Marine Science 43:710-729.

DEMERS, S., LEGENDRE, L., and THERRIAULT, J.-C. 1986. Phytoplankton responses to vertical tidal mixing. In: Bowman, J., Yentsch, M., and Peterson, W.T., eds. Tidal mixing and plankton dynamics. Berlin: Springer-Verlag. 1-40.

DEMERS, S., THERRIAULT, J.-C., BOURGET, E., and BAH, A. 1987. Resuspension in the shallow sublittoral zone of a macrotidal estuarine environment: Wind influence. Limnology and Oceanography 32:327-339.

DUGDALE, R.C. 1972. Chemical oceanography and primary productivity in upwelling regions. Geoforum 11:47-61.

EPPLEY, R.W., and COATSWORTH, J.L. 1966. Culture of the marine phytoplankter, Dunaliella tertiolecta, with light-dark cycles. Archiv fur Mikrobiologie 55:66-80.

FALKOWSKI, P.G. 1983. Light-shade adaptation and vertical mixing of marine phytoplankton: A comparative field study. Journal of Marine Research 41:215-237.

1984. Physiological responses of phytoplankton to natural light regimes. Journal of Plankton Research 6:295-307.

GABRIC, A.J., and PARSLOW, J. 1989. Effect of physical factors on the vertical distribution of phytoplankton in eutrophic coastal waters. Australian Journal of Marine Freshwater Research 40:559-569.

GLIBERT, P.M., and GOLDMAN, J.C. 1981. Rapid ammonium uptake by marine phytoplankton. Marine Biology Letters 2:25-31.

GRAINGER, E.H. 1975. A marine ecology study in Frobisher Bay, arctic Canada. In: Cameron, T.W.M., and Billingsley, L.W., eds. Energy flow its biological dimension. Ottawa: The Royal Society of Canada. 261-266.

HARDING, L.W., Jr., and HEINBOKEL, J.F. 1984. Periodicities of photosynthesis and cell division: Behavior of phase-lagged replicate cultures of Ditylum brightwellii in a diurnally varying photic regime. Marine Ecology - Progress Series 15:225-232.

HARRIS, G.P., and TRIMBEE, A.M. 1986. Phytoplankton population dynamics of a small reservoir: Physical/biological coupling and the time scales of community change. Journal of Plankton Research 8:1011-1025.

HAURY, L.R., WIEBE, P.H., ORR, M.H., and BRISCOE, M.G. 1983. Tidally generated high-frequency internal wave packets and their effects on plankton in Massachusetts Bay. Journal of Marine Research 41:65-112.

HELLEBUST, J.A., and LEWIN, J. 1977. Heterotrophic nutrition. In: Werner, D., ed. The biology of diatoms. Oxford: Blackwell Scientific Publications. 169-197.

HOLLIGAN, P.M. 1978. Patchiness in subsurface phytoplankton populations on the northwest European continental shelf. In: Steele, J.H., ed. Spatial pattern in plankton communities. New York: Plenum Press. 221-238.

HSIAO, S.I.C. 1980. Quantitative composition, distribution, community structure and standing stock of sea ice microalgae in the Canadian Arctic. Arctic 33:768-793.

1985. The growth of arctic marine phytoplankton in Frobisher Bay. Arctic 38:31-38.
1988. Spatial and seasonal variations in primary production of sea ice microalgae and phytoplankton in Frobisher Bay, arctic Canada. Marine Ecology - Progress Series 44:275-285.

HSIAO, S.I.C., FOY, M.G., and KITTLE, D.W. 1977. Standing stock, community structure, species composition, distribution, and primary production of natural populations of phytoplankton in the southern Beaufort Sea. Canadian Journal of Botany 55:685-694.

HULBURT, E.M. 1963. The diversity of phytoplanktonic populations in oceanic, coastal and estuarine regions. Journal of Marine Research 21:81-93.

IBANEZ, F. 1971. Effet des transformations des données dans l'analyse factorielle en écologie planctonique. Cahier océanographie 23:545-561.

IGNATIADES, L. 1969. Annual cycle, species diversity and succession of phytoplankton in lower Saronicos Bay, Aegean Sea. Marine Biology 3:196-200

1979. The influence of water stability on the vertical structure of a phytoplankton community. Marine Biology 52:97-104.

IVERSON, R.L., CURL, H.C., Jr., O'CONNORS, H.B., Jr., KIRK, D., and ZAKAR, K. 1974. Summer phytoplankton blooms in Auke Bay, Alaska, driven by wind mixing of the water column. Limnology and Oceanography 19:271-278.

JEFFREY, S.W., and HUMPHREY, G.F. 1975. New spectrophotometric equations for determining chlorophylls $\mathrm{a}, \mathrm{b}, \mathrm{cl}$ and $\mathrm{c} 2$ in higher plants, algae and natural phytoplankton. Biochemie und Physiologie der Pflanzen (BPP) 167:191-194.

KAMYKOWSKI, D. 1981. Laboratory experiments on the diurnal vertical migration of marine dinoflagellates through temperature gradients. Marine Biology 62:57-64.

KANA, T.M., WATTS, J.L., and GLIBERT, P.M. 1985. Diel periodicity in the photosynthetic capacity of coastal and offshore phytoplankton assemblages. Marine Ecology - Progress Series 25:131-139.

KAWARADA, Y., and SANO, A. 1968. Diel variations of phytoplankton, chlorophyll a and photosynthesis. Bulletin of the Plankton Society of Japan 15:7-10.

KIEFER, D.A., and KREMER, J.N. 1981. Origins of vertical patterns of phytoplankton and nutrients in the temperate, open ocean: A stratigraphic hypothesis. Deep-Sea Research 28A:1087-1105.

LANDE, R., and YENTSCH, C.S. 1988. Internal waves, primary production and compensation depth of marine phytoplankton. Journal of Plankton Research 10:565-571.

LANGDON, C. 1988. On the causes of interspecific differences in the growth-irradiance relationship for phytoplankton. II. A general review. Journal of Plankton Research 10:1291-1312.

LEGENDRE, L., DEMERS, S., GARSIDE, C., HAUGEN, E.M., PHINNEY, D.A., SHAPIRO, L.P., THERRIAULT, J.-C., and YENTSCH, C.M. 1988. Circadian photosynthetic activity of natural marine phytoplankton isolated in a tank. Journal of Plankton Research 10:1-6.

LEGENDRE, L., INGRAM, R.G., and SIMARD, Y. 1982. Aperiodic changes of water column stability and phytoplankton in an arctic coastal embayment, Manitounuk Sound, Hudson Bay. Naturaliste Canadien 109:775786.

LEVASSEUR, M., THERRIAULT, J.-C., and LEGENDRE, L. 1984. Hierarchical control of phytoplankton succession by physical factors. Marine Ecology - Progress Series 19:211-222.

LEWIN, J., and RAO, V.N.R. 1975. Blooms of surf-zone diatoms along the coast of the Olympic Peninsula, Washington. V. Daily periodicity phenomena associated with Chaetoceros armatum in its natural habitat. Journal of Phycology 11:330-338.

LEWIN, J.C., REIMANN, B.E., BUSBY, W.F., and VOLCANI, B.E. 1966 Silica shell formation in synchronously dividing diatoms. In: Cameron, I.L., and Padilla, G.M., eds. Cell synchrony - studies in biosynthetic regulation. New York: Academic Press. 169-188.

LITAKER, W., DUKE, C.S., KENNEY, B.E., and RAMUS, J. 1987. Shortterm environmental variability and phytoplankton abundance in a shallow tidal estuary. I. Winter and summer. Marine Biology 96:115-121.

MARGALEF, R. 1977. Ecosystem diversity differences: Poles and tropics. In: Dunbar, M.J., ed. Polar oceans. Calgary: Arctic Institute of North America. 367-375.

1978. Diversity. In: Sournia, A., ed. Phytoplankton manual. Paris The United Nations Educational, Scientific and Cultural Organization. 251-260.

MARRA, J., and HEINEMANN, K. 1982. Photosynthesis response by phytoplankton to sunlight variability. Limnology and Oceanography 27:11411153.

NELSON, D.M., and BRAND, L.E. 1979. Cell division periodicity in 13 species of marine phytoplankton on a light:dark cycle. Journal of Phycology 15:67-75. 
ODUM, E.P. 1971. Fundamentals of ecology. 3rd ed. Philadelphia: W.B. Saunders Co. $574 \mathrm{p}$.

OWENS, T.G., FALKOWSKI, P.G., and WHITLEDGE, T.E. 1980. Diel periodicity in cellular chlorophyll content in marine diatoms. Marine Biology 59:71-77.

PIELOU, E.C. 1975. Ecological diversity. New York: John Wiley \& Son. 165 p.

PINGREE, R.D., HOLLIGAN, P.M., and MARDELL, G.T. 1978. The effects of vertical stability on phytoplankton distributions in the summer on the northwest European Shelf. Deep-Sea Research 25:1011-1028.

PIRSON, A., and LORENZEN, H. 1966. Synchronized dividing algae. Annual Review of Plant Physiology 17:439-458.

RAYMONT, J.E. 1980. Plankton and productivity in the oceans. 2nd ed. Vol. 1, Phytoplankton. Oxford: Pergamon Press. 489 p.

REED, C. 1978. Species diversity in aquatic microecosystems. Ecology 59:481-488

REID, F.M.H., STEWART, E., EPPLEY, R.W., and GOODMAN, D. 1978. Spatial distribution of phytoplankton species in chlorophyll maximum layers off southern California. Limnology and Oceanography 23:219-226.

SAS INSTITUTE. 1988. SAS/STAT user's guide. Release 6.03 edition. Cary, North Carolina: SAS Institute Inc.

SHANNON, C.E., and WEAVER, W. 1949. The mathematical theory of communication. Urbana: University of Illinois. $125 \mathrm{p}$.

SHIMADA, B.M. 1958. Diurnal fluctuation in photosynthetic rate and chlorophyll " $a$ " content of phytoplankton from eastem Pacific waters. Limnology and Oceanography 3:336-339.

SILVA, P.C. 1980. Names of classes and families of living algae. Regnum Vegetabile 103:1-156.

SOEDER, C.J. 1965. Some aspects of phytoplankton growth and activity. In: Goldman, C.R., ed. Primary productivity in aquatic environments. Berkeley: University of California Press. 47-59.

SOURNIA, A. 1974. Circadian periodicities in natural populations of marine phytoplankton. Advances in Marine Biology 12:325-389.

STEELE, J.H., and YENTSCH, C.S. 1960. The vertical distribution of chlorophyll. Journal of the Marine Biological Association of the United Kingdom 39:217-226.

STEEMANN NIELSEN, E., and JORGENSEN, E.G. 1962. The physiological background for using chlorophyll measurements in hydrobiology and a theory explaining daily variations in chlorophyll concentration. Archiv fur Hydrobiologie 58:349-357.
STRICKLAND, J.D.H., and PARSONS, T.R. 1972. A practical handbook of seawater analysis. Fisheries Research Board of Canada Bulletin 167:1-310.

SWIFT, E., 5th. 1967. Cleaning diatom frustules with ultraviolet radiation and peroxide. Phycologia 6:161-163.

TISELIUS, P. 1988. Effects of diurnal feeding rhythms, species composition and vertical migration on the grazing impact of calanoid copepods in the Skagerrak and Kattegat. Ophelia 28:215-230.

TURPIN, D.H., and HARRISON, P.J. 1979. Limiting nutrient patchiness and its role in phytoplankton ecology. Journal of Experimental Marine Biology and Ecology 39:151-166.

VANDEVELDE, T., LEGENDRE, L., DEMERS, S., and THERRIAULT, J.-C. 1989. Circadian variations in photosynthetic assimilation and estimation of daily phytoplankton production. Marine Biology 100:525-531.

VANDEVELDE, T., LEGENDRE, L., THERRIAULT, J.-C., DEMERS, S., and BAH, A. 1987. Sub-surface chlorophyll maximum and hydrodynamics of the water column. Journal of Marine Research 45:377-396.

VENRICK, E.L. 1988. The vertical distributions of chlorophyll and phytoplankton species in the North Pacific central environment. Journal of Plankton Research 10:987-998.

WANGERSKY, P.J. 1977. The role of particulate matter in the productivity of surface waters. Helgolander Wissenschaftliche Meeresuntersuchungen 30:546-564.

WEILER, C.S. 1980. Population structure and in situ division rates of Ceratium in oligotrophic waters of the North Pacific central gyre. Limnology and Oceanography 25:610-619.

WEILER, C.S., and CHISHOLM, S.W. 1976. Phased cell division in natural populations of marine dinoflagellates from shipboard cultures. Journal of Experimental Biology and Ecology 25:239-247.

WHALEN, S.C., and ALEXANDER, V. 1984. Diel variations in inorganic carbon and nitrogen uptake by phytoplankton in an arctic lake. Journal of Plankton Research 6:571-590.

WHITLEDGE, T.E., and WIRICK, C.D. 1983. Observations of chlorophyll concentrations of Long Island from a moored in situ fluorometer. DeepSea Research 30:297-309.

WILLIAMSON, C.E. 1980. Phased cell division in natural and laboratory populations of marine plankton diatoms. Journal of Experimental Biology and Ecology 43:271-279.

WOOD, E.J.F., and CORCORAN, E.F. 1966. Diurnal variation in phytoplankton. Bulletin of Marine Science 16:383-403. 